EESTI NSV TEADUSTE AKADEEMIA TOIMETISED. 29. KOIDE FOOSIKA * MATEMAATIKA. 1980, NR. 2

ИЗВЕСТИЯ АКАДЕМИИ НАУК ЭСТОНСКОИ ССР. ТОМ 29 ФИЗИКА - МАТЕМАТИКА. 1980, № 2

А. ФРЕИБЕРГ, А. РАЙДАРУ, А. АНИЯЛГ, К. ТИМПМАНН, П. КУКК, П. СААРИ

\title{
СИСТЕМА НЕПРЕРЫВНОЙ ВРЕМЕННОЙ РАЗВЕРТКИ ОПТИЧЕСКИХ СПЕКТРОВ НА ОСНОВЕ ЭЛЕКТРОННО-ОПТИЧЕСКОГО ПРЕОБРАЗОВАТЕЛЯ, РАБОТАЮЩЕГО СИНХРОННО С ПИКОСЕКУНДНЫМ ЛАЗЕРОМ
}

\author{
(Представил К. К. Ребане)
}

\section{1. Введение}

Времяанализирующие электронно-оптические преобразователи (ЭОП) являются в настоящее время единственными приборами, позволяющими проводить прямые временные измерения оптических процессов с пикосекундным разрешением $\left[{ }^{1-3}\right]$. До сих пор для генерации ультракоротких световых импульсов использовались в основном мощные твердотельные лазеры с пассивной синхронизацией мод. Недостатками этих лазеров являются низкая частота повторения и невысокая стабильность пиковой мощности импульсов. В последнее время все большее распространение получают синхронно накачиваемые непрерывные лазеры на красителе, генерирующие стабильные пико- и субпикосекундные импульсы с весьма высокой частотой ( 100 МГц) повторения. При этом мощность одиночного импульса относительно мала ( $~ 100-$ $1000 B T$ ), что во многих случаях чрезвычайно выгодно, например, при исследовании объектов, склонных к фоторазрушению, а также при необходимости предотвратить побочные нелинейные эффекты. По нашему мнению, пикосекундная спектроскопия на базе таких лазеров и соответствующей регистрирующей аппаратуры перспективна и для исследования ранних стадий спонтанного (резонансного) вторичного свечения. Теоретические основы таких исследований, позволяющие детально изучать процессы преобразования световой энергии в кристаллах и молекулах, разрабатываются в последнее время весьма интенсивно [4-11]. Однако слабость импульса вынуждает многократно накапливать и усреднять результаты отдельных измерений. При работе с ЭОП это достигается периодической (с частотой строго равной или кратной частоте повторения импульсов лазера) разверткой изображения.

Первые работы с использованием принципа непрерывной периодической (как линейной, так и круговой) развертки ЭОП были выполнены еще в 50 -е годы $\left[{ }^{12,13}\right]$. Однако в этих работах высокочастотная развертка не была синхронизована с изучаемым явлением, отчего теряла бо́льшую часть своих преимуществ перед обычно используемой 
однократной импульсной разверткой. Синхронная развертка была впервые реализована в $\left[{ }^{14}\right]$. В $\left[{ }^{15}\right]$ этот метод применялся для изучения коротких световых импульсов инжекционных полупроводниковых лазеров. В последнее время в связи с освоением синхронно накачиваемых непрерывных лазеров на красителе появились новые работы $\left[{ }^{18-18}\right]$, посвященные разработке систем синхронной развертки ЭОП. При этом авторами $\left[{ }^{16,17}\right]$ с однокамерным ЭОП «Photochron 1» достигнуто временное разрешение $\leqslant 10$ псек.

В данной работе описана разработанная нами на базе однокамерного ЭОП ПИМ-ЗШ (фотокатод-кислородно-серебряно-цезиевый) и четырехкамерного ЭОП УМИ-93Ш (фотокатод-многощелочной) система непрерывной синусоидальной временной развертки, работающая синхронно с пикосекундным лазером на красителе с частотой повторения импульсов $82 M Г u$. Система отличается относительной простотой исполнения и высокой надежностью работы.

\section{2. Экспериментальная установка}

Основой экспериментальной установки (см. рис. 1) служат ЭОП, комплексная лазерная система фирмы «Спектра-Физикс» $(\mathrm{Kr}+$-лазер модели 171 с активной синхронизацией мод оптикоакустическим модулятором модели 342/452 и лазер на красителе модели 375) и система синхронной развертки. Последнюю можно условно разбить на три части:

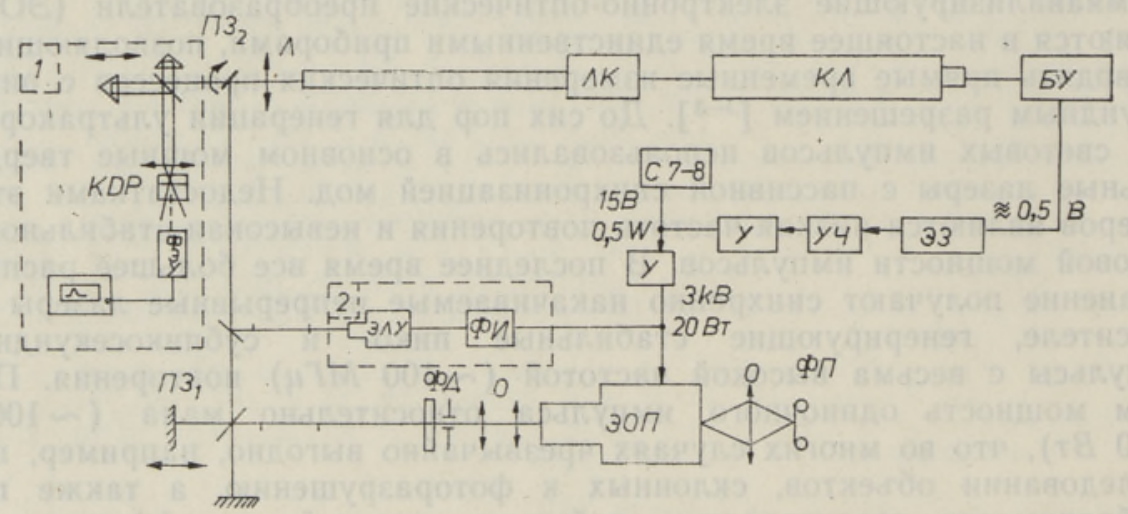

Рис. 1. Схема экспериментальной установки. Обозначения: КЛ $-\mathrm{Kr}^{+}$-лазер, ЛК - лазер на красителе, БУ - блок управления оптикоакустическим модулятором, уч - удвонтель частоты, $\mathbf{y}-$ усилитель, Э3- узел электрической задержки, Л - линза, О - объектив, ПЗ - полупрозрачное зеркало, $\Phi$ - фильтр, Д - диафрагма, ФП - фотоприставка. Отдельно выделены: $I$ - автокоррелятор и 2 - система импульсной развертки ЭОП.

удвоитель частоты, предусилитель и усилитель мощности. Высокочастотное развертывающее напряжение, достигающее на пластинах ЭОП $\sim 3 \kappa B$, подается только на одну пару пластин. Синхронность развертки с работой лазера обеспечивается тем, что отклоняющее напряжение подается (после удвоения по частоте и усиления приблизительно в $3 \cdot 10^{3}$ раз) непосредственно из блока управления оптикоакустическим модулятором лазера (см. рис. 1). Режим системы синхронной раззертки проверялся стробоскопическим осциллографом С7-8. Перемещение наблюдаемого изображения по временной оси экрана ЭОП и в 
центр экрана, где разрешение наилучшее, осуществлялось узлом электрической задержки, представляющей собой набор кусков коаксиального кабеля разной длины. Стационарная картина с экрана ЭОП регистрировалась фотографированием через светосильный объектив $(\mathrm{F} / 1,5$, 8,5 cм) на фотопленку РФ-3, которая затем микрофотометрировалась на приборе ИФО-451. Перестановка системы синхронной развертки с ПИМ-ЗШ на УМИ-93Ш требует лишь перенастройки высоковольтного триммера.

\section{3. Результаты испытания системы и обсуждение}

Испытание системы на временное разрешение проводилось импульсами синхронно накачиваемого непрерывного лазера на оксазиновом красителе (пределы генерации 690-805 нм), который в режиме многочастотной генерации $\left[{ }^{19}\right]$ выдает импульсы длительностью от 5 до 10 псек. Длительность определялась независимым образом по автокорреляционной функции интенсивности, которая регистрировалась по схеме неколлинеарной генерации второй гармоники в кристалле KDP $\left[{ }^{19}\right]$ (рнс. 1). Характерная для используемых в данной работе импульсов автокорреляционная функция показана на рис. 2.

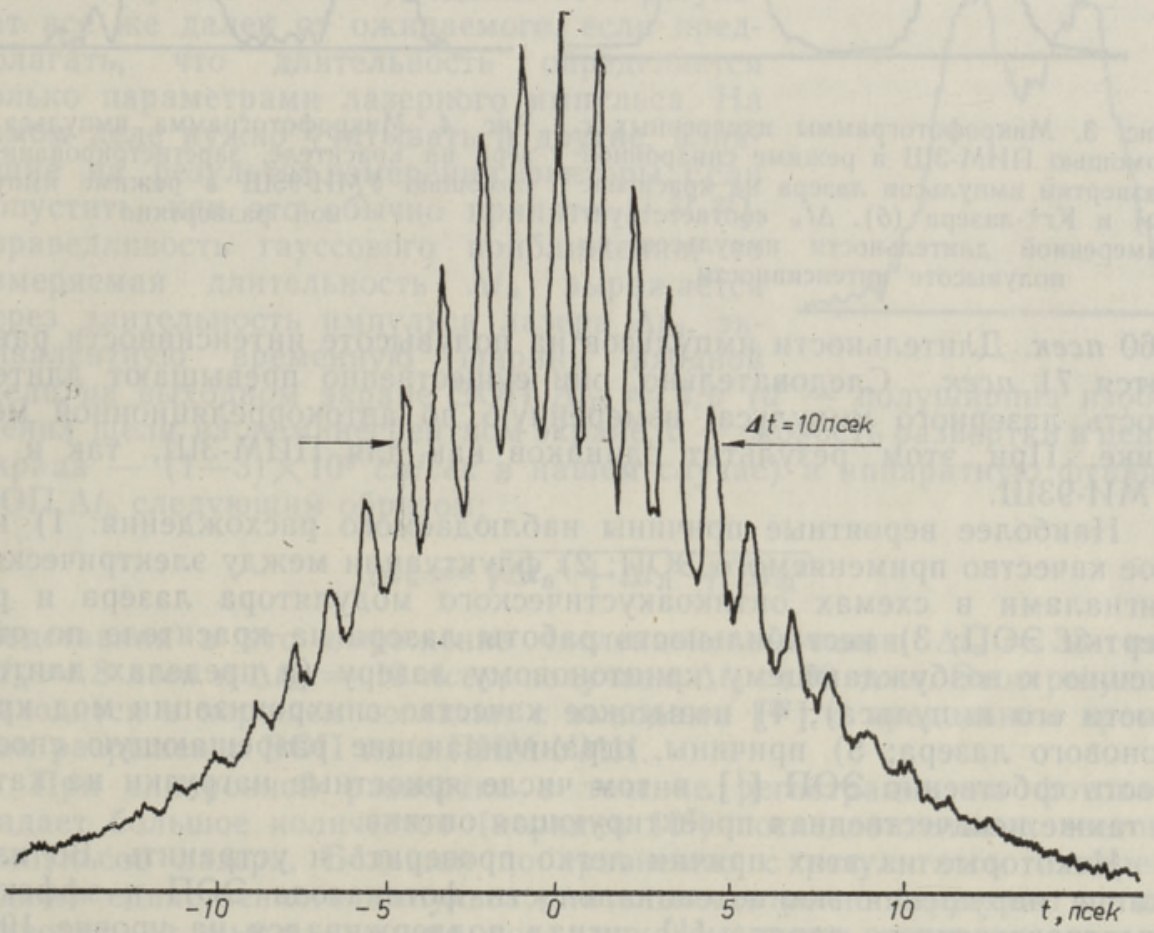

Рис. 2. Пример автокорреляционной функции используемых пикосекундных импульсов лазера на красителе.

На рис. 3,a приведена типичная микрофотограмма двух световых импульсов лазера на оксазине, полученных разделением каждого выходящего из лазера импульса на полупрозрачном зеркале ПЗ (рис. 1). Временной интервал между импульсами, регулируемый в широких пределах системой оптической задержки, в данном случае составлял 


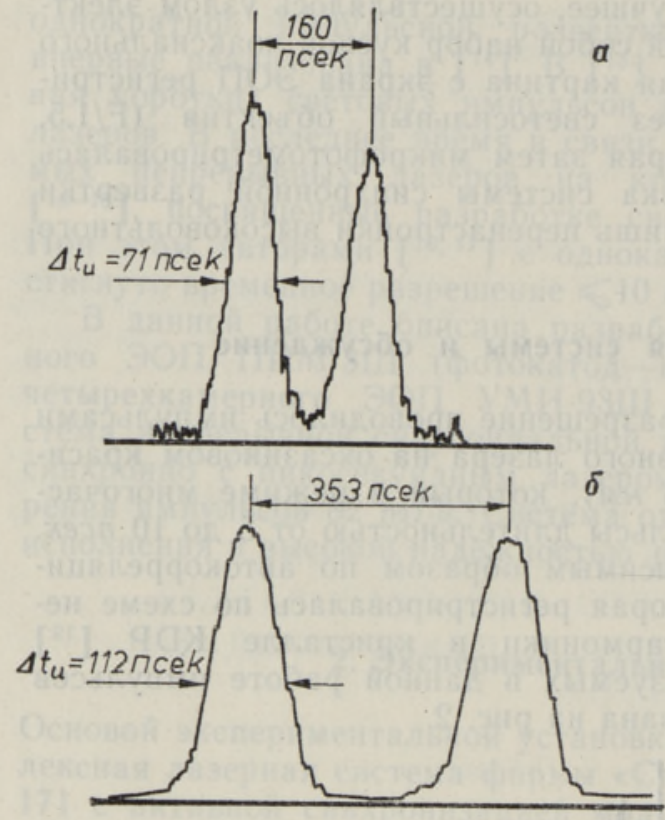

Рис. 3. Микрофотограммы измеренных с помощью ПИМ-ЗШ в режиме синхронной развертки импульсов лазера на красителе (a) и $\mathrm{Kr}^{+}$-лазера (б). $\Delta t_{u}$ соответствует измеренной длительности импульсов на полувысоте интенсивности.

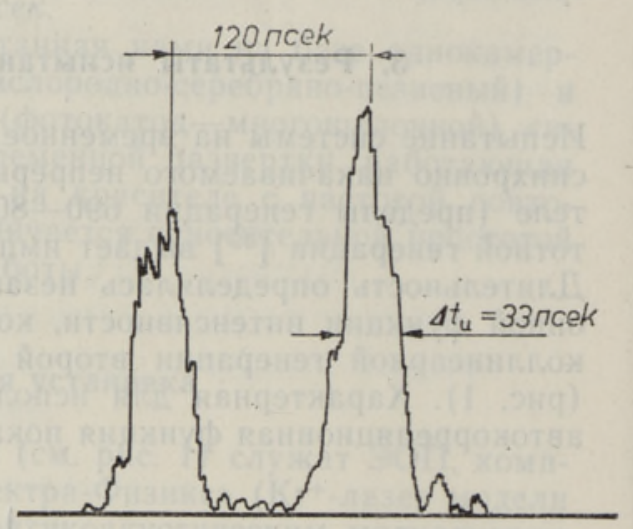

Рис. 4. Микрофотограмма импульса лазера на красителе, зарегистрированная с помощью УМИ-93Ш в режиме импульсной развертки.

160 псек. Длительности импульсов на полувысоте интенсивности равняются 71 псек. Следовательно, они существенно превышают длительность лазерного импульса, измеренную по автокорреляционной методике. При этом результат одинаков как для ПИМ-ЗШ, так и для УМИ-93Ш.

Наиболее вероятные прнчины наблюдаемого расхождения: 1) низкое качество применяемого ЭОП; 2) флуктуации между электрическими сигналами в схемах оптикоакустического модулятора лазера и развертки ЭОП; 3) нестабильность работы лазера на красителе по отношению к возбуждающему криптоновому лазеру (в пределах длительности его импульса); 4) невысокое качество синхронизации мод криптонового лазера; 5) причины, ограничивающие разрешающую способность собственно ЭОП [ $\left.{ }^{1}\right]$, в том числе яркостные нагрузки на катод, а также некачественная проектирующая оптика.

Некоторые из этих причин легко проверить и устранить. Во избежание нарушения эквипотенциальности фотокатода ЭОП и эффектов пространственного заряда $\left.{ }^{1}{ }^{1}\right]$ сигнал поддерживался на уровне 100 1000 фотоэлектронов с разрешенного элемента катода. Качество проектирующей оптики, установленной перед ЭОП (система из двух объективов Юпитер-9 (F/2, 8,5 cM) с передачей изображения $1: 1)$, и статическое пространственное разрешение ЭОП проверялись визуально стандартными мирами. Разрешение ПИМ-3Ш 53 nл/мм (ускоряющее напряжение $15 \kappa B)$, УМИ-93Ш - $30 \mathrm{n} \Omega /$ мм $(15,10,10,10 \kappa B)$.

О качестве синхронизации мод криптонового лазера можно судить по длительности его импульсов. Как следует из рис. 3,б, с учетом временного разрешения нашей системы (см. дальше), длительность им- 
Рис. 5. Влияние дрожания импульсов на временное разрешение ЭОП (микрофотограмма): за время регистрации интегрированы $\sim 10^{7}(a)$ и $\sim 1400$ импульсов лазера (б).

пульсов $\mathrm{Kr}^{+}$-лазера равняется $\sim 90$ псек, что свидетельствует о неплохом качестве синхронизации мод $\left[{ }^{16}\right]$.

Гораздо труднее выявить роль первых трех причин. Для определения аппаратной функции ЭОП (т. е. результата реакции ЭОП на бесконечно короткий световой импульс) мы развертывали одиночный импульс лазера на красителе с помощью аппаратуры линейной импульсной развертки (длительность развертки $\sim 7$ нсек), аналогичной описанной в $\left[{ }^{20}\right]$ (см. рис. 4). При сравнении рис. 4 с рис. $3, a$ обнаруживается существенное сокращение длительности регистрируемого импульса (33 псек против 71 псек), однако этот результат все же далек от ожидаемого, если предполагать, что длительность определяется только параметрами лазерного импульса. На самом деле нужно учитывать и другие, влияющие на результат измерения, факторы. Если допустить, как это обычно принято $[1,21,22]$, справедливость гауссового приближения, то измеряемая длительность $\Delta t_{u}$ выражается через длительность импульса лазера $\Delta t_{\text {л }}$, эквивалентную временную ширину входной

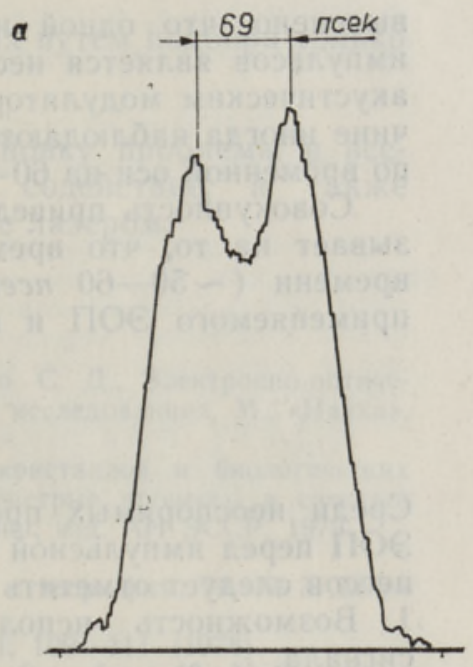
щели на выходном экране ЭОП $\Delta t_{\text {щ }}=d_{/}^{\prime} v(d-$ полуширина изображения щели на люминесцентном экране, $v$-скорость развертки в центре экрана - $(1-3) \times 10^{9}$ см/сек в нашем случае) и аппаратную функцию ЭОП $\Delta t_{\mathrm{a}}$ следующим образом:

$$
\Delta t_{u}=\sqrt{\Delta t_{\mathrm{a}}{ }^{2}+\Delta t_{\mathrm{s}}{ }^{2}+\Delta t_{\mathrm{m}}{ }^{2}}
$$

Подставляя в это выражение численные значения $\Delta t_{u}=33$ nсек, $\Delta t_{\mathrm{I}}=8$ nсек и $\Delta t_{\text {щ }}=10$ псек, получаем $\Delta t_{\mathrm{a}} \simeq 30$ псек. Этот результат находится в хорошем согласии с выводами $\left[{ }^{22}\right]$ о предельном временном разрешении ЭОП типа ПИМ-УМИ.

При синхронной развертке в течение регистрации на фотокатод падает большое количество (порядка $10^{7}$ ) последовательных световых импульсов лазера. Бо́льшая по сравнению с результатом измерения одного единственного импульса длительность регистрируемого сигнала наводит на мысль, что не все импульсы точно синхронизованы во времени (импульсы дрожат друг относительно друга). Для проверки этого предположения мы сравнили разрешения ӘОП при разных длительностях экспонирования фотокатода. В качестве прерывателя использовалось вращающееся зеркало $\Pi_{2}$ (рис. 1). Изменение экспози. ции достигалось изменением скорости вращения зеркала и надлежащим фокусированием луча. Как видно из рис. 5, если на фотокатод падает $\sim 1400$ импульсов вместо $\sim 10^{7}$, действительно, наблюдается некоторое улучшение временного разрешения. Тщательное изучение данного круга вопросов продолжается, однако к настоящему моменту 
выяснено, что одной из основных причин асинхронности отдельных импульсов является нестабильность работы блока управления оптикоакустическим модулятором $\mathrm{Kr}^{+}$-лазера. По-видимому, по этой же причине иногда наблюдаются случайные скачки импульсов на экране ЭОП по временной оси на $60-80$ псек.

Совокупность приведенных фактов, на наш взгляд, однозначно указывает на то, что временное разрешение, достигнутое к настоящему времени $(\sim 50-60$ псек), определяется в основном несовершенством применяемого ЭОП и невысокой стабильностью работы лазеров.

\section{4. Заключение}

Среди неоспоримых преимуществ непрерывной синхронной развертки ЭОП перед импульсной разверткой в исследованиях сверхбыстрых процессов следует отметить следующие.

1. Возможность использования современных методов накоплення сигнала.

2. Простота юстировогных и калибровочных операций благодаря стационарности сигнала на выходном экране ЭОП; существенное сокращение времени измерения, поскольку отпадает необходимость в пробных фотографированиях.

3. Отсутствие проблем, связанных с неконтролируемым собственным временем срабатывания электронной схемы развертки.

4. Возможность сравнительно просто с помощью фотоэлектрических датчиков автоматизировать весь процесс измерений и обработки, не прибегая к сложным и дорогостоящим высокоскоростным аналого-цифровым преобразователям и к промежуточной памяти большого объема.

5. Высокая надежность работы и простота использования.

Из недостатков данной системы прежде всего следует отметить нелинейность развертки и некоторую ограниченность диапазона измеряемых временных процессов (определяется интервалом между последовательными импульсами). Однако эти недостатки не принципиальны. Нелинейность развертки может быть легко учтена или автоматически исправлена с помощью ЭВМ, особенно при фотоэлектрической регистрации информации. Интервал в 12 нсек между последовательными импульсами обычно вполне достаточен для исследования процессов в пико- и субнаносекундной областях.

Реализация всех перечисленных выше преимуществ требует перехода от фотографической регистрации к фотоэлектрической. На наш взгляд, перспективен вариант с телевизионным регистрирующим устройством на видиконе, соединенным с ЭВМ. Существенное улучшение временного разрешения системы предполагает также использование более качественного ЭОП и обеспечение высокой стабильности работы лазера. Тем не менее, как показали первые измерения времяразрешенных спектров в начатом цикле исследования переходных спектров вторичного свечения, данная система позволяет успешно решать задачи спектрохронографирования слабого оптического сигнала в субнаносекундном диапазоне.

В заключение добавим, что синхронно накачиваемый лазер на красителе, работающий в устойчивом режиме одновременной генерации пикосекундных импульсов разной длины волны $\left[{ }^{19}\right]$, дает возможность прецизионного исследования пико- и субпикосекундных временных характеристик ЭОП. Согласно $\left[{ }^{19}\right]$, временные характеристики генерируемых этим лазером ультракороткнх импульсов с периодической (суб)- 
пикоструктурой регулируемы в широких пределах путем подбора только селекторов-эталонов.

Авторы благодарны К. К. Ребане за постановку проблемы и всестороннюю помощь, Л. Ребане за ценное содействие, а также Р. Каарли и Я. Аавиксоо за помощь при работе с лазером.

\section{ЛИТЕРАТУРА}

1. Бутслов М. М,, С тепанов Б. М., Фанченко С. Д., Электронно-оптические преобразователи и их применение в научных исследованиях, М., «Наука», 1978.

2. Пикосекундные методы в спектроскопии молекул, кристаллов и биологических систем. (Мат-лы междунар. симпознума «Сверхбыстрые процессы в спектроскопин», Таллин, 27 сент. - 1 окт. 1978 г.), Таллин, изд. АН ЭССР, 1979

3. Шелев М. Я., там же, с. $84-97$.

4. Хи жняков В. В., Р еб а пе И. К., Ж. эксперим. и теор. физ., 74, № 3, 885896 (1978).

5. S a a r i, P., ENSV TA Toimet., Füüs. Matem., 27, № 1, 109-111 (1978)

6. Toyoz a wa, Y., Kotani, A., Sumi, A., J. Phys. Soc. Jap., 42, № 5, 14951505 (1977).

7. Kubo, R., Takag a hara, T., H a n mura, E., ibid., 43, № 5, 1522-1532 (1977).

8. Ребане И. К., Ту ул А. Л., Хи жняков В. В., Ж. эксперим. и теор. физ., 77, № 4, 1302-1312 (1979).

9. R e b a ne, K. K., J. Luminescence, 18/19, 693-699 (1979).

10. Rebane, K. K., In: Ultrafast Relaxation and Secondary Emission (proc. of thie Intern. Symposium «UItrafast Phenomena in Spectroscopy», Tallinn, Sept. 27 Oct. 1, 1978), Tallinn, ed. by Estonian SSR Acad. Sci., 1979, p. 7-37.

11. S a a ri, P. In: Light Scattering in Solids, ed. by Birman, J. L., Cummins, H. Z., Rebane, K. K., Plenum Press, New York-London, 1979, p. 315-329.

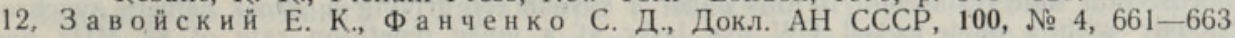
(1955).

13. Болотин В. Ф., Демидов Б. А., Завойский Е. К., Смолкин Г. Е., Ск а чков Ю. Ф., Фан ченко С. Д., Успехи науч. фотогр., 19, 175 (1964).

14. Зинин Э. И., Коробейииков Л. С., Кулипанов Г. Н., Л аза рен ко Б. Л., М а т еев Ю. Т., Попов С. Г., Скринский А. Н., Ст а о д у 6 це в Т. П., Т у л айко в Г. М., Атом. энергия, 20, № 3, 320-324 (1966).

15. Федоров В. Б., Новиков А. А., Ш а рыпин А. А., Приборы и техн. эксперимента, № 5, 142-145 (1969).

16. A d a m s, M. C., S i b b et t, W., B r a d l e y, D. J., Opt. Commun., 26, № 2, $273-276$ (1978).

17. T a ylor, J. R., A d a m s, M. C., S ibbett, W., Appl. Phys. Lett., 35, № 8, $590-592$ (1979).

18. Berndt, K., Klose, E., In: Picosecond Methods in Spectroscopy of Molecules, Crystals and Biological Systems (proc. of the Intern. Symposium \&Ultrafast Phenomena in Spectroscopy», Tallinn, Sept. 27 - Oct. 1, 1978), Tallinn, ed. by Estonian SSR Acad. Sci., 1979, p. 129-134.

19. К а а рли Р. К., А а иксооЯ. Ю., В илл А. А., Кукк П. Л., С а а и П. М., $Ф$ рейберг А. М., Квант. электроника, 6, № 12, 2630-2633 (1979).

20. Коробкин В. В., Сердюченко Ю. Н., Щелев М. Я., В кн.: Науч. тр. ВНиИОФИ, Электронно-оптические преобразователи и их применение в науке и технике, Сер. В, вып. I, М., 1972, с. 166-173.

21. B r a d le y, D. J., N e w, G. H. C., Proc. IEEE, 62, № 3, 313--345 (1974).

22. Коробкин В. В., Малютин А. А., Щелев М. Я., Ж. техн. физ., 41, № 1 , $216-219(1971)$.

Ннститут физики

Академии наук Эстонской ССР
Поступила в редакцию

$21 /$ II 1980 
A. FREIBERG, A. RAIDARU, A. ANIJALG,

K. TIMPMANN, P. KUKK, P. SAARI

\section{OLIVÄLKLASERIGA SUNKRONISEERITUD ELEKTRONOPTILISEL MUUNDURIL PÖHINEV OPTILISTE SPEKTRITE PIDEVA AEGLAOTUSE SUSTEEM}

Aeganalüüsivad elektronoptilised muundurid võimaldavad otseselt mõõta optiliste protsesside ajalist kulgu pikosekundilise eristustäpsusega. Käesolevas töös on muundurite УМИ-93Ш ја ПИМ-ЗШ baasil loodud aparatuur optilise signaali pidevaks ajaliseks laotamiseks sünkroonselt sagedusel $82 \mathrm{MHz}$ töötava ülivälklaseri kiirgusimpulssidega. Aeglaotuse sünkroniseerimiseks kasutatakse vahetult laseri.akusto-optilise muunduri tüürploki väljundsignaali. Süsteemi ajalise eristustäpsuse $(\approx 50$ ps $)$ määravad kasutatavad muundurid ja laseri ebastabiilsus.

Artiklis on analüüsitud mõningaid eeliseid, mida omab ülikiirete valgusnähtuste uurimisel pidev aeglaotus üksikimpulsslaotusega võrreldes. Esialgsete katsete kohaselt saab süsteemi edukalt kasutada nỗrga valgussignaali aeglaotuseks subnanosekund-diapasoonis.

A. FREIBERG, A. RAIDARU, A. ANIJALG,

K. TIMPMANN, P. KUKK, P. SAARI

\section{STREAK CAMERA OPERATING IN SYNCHRONISM WITH A PICOSECOND LASER FOR REAL-TIME OPTICAL PROCESS MEASUREMENT}

Electro-optical streak cameras admit of a direct measurement of optical phenomena with picosecond time resolution. In this paper a system for driving a streak camera synchronously with a mode-locked $\mathrm{CW}$ dye laser at a pulse repetition rate of $82 \mathrm{MHz}$ has been elaborated and described. Part of the signal directed to the acousto-optic modelocking prism of the pumping $\mathrm{Kr}^{+}$-laser passes through the frequency-doubling and amplification (up to $3 \mathrm{kV}$ ), and is used for a synchronous driving of the streak tube deflection. The time resolution achieved by the present time $(\sim 50 \mathrm{ps})$ is limited by the streak tubes used and by the mode-locking instabilities of the laser.

Some advantages of the synchronous driving of a streak camera over the commonly used pulsed deflection are discussed. Preliminary experiments indicate the reliability and perspectivity of our arrangement for measuring low-signal optical phenomena in the subnanosecond time region. 